\title{
Automatic Image Registration Using Mexican Hat Wavelet, Invariant Moment, and Radon Transform
}

\author{
Jignesh N Sarvaiya \\ Electronics Engg. Dept \\ S V National Institute of Technology \\ Surat, India
}

\author{
Dr. Suprava Patnaik \\ Electronics Engg. Dept \\ S V National Institute of Technology \\ Surat, India
}

\begin{abstract}
Image registration is an important and fundamental task in image processing used to match two different images. Given two or more different images to be registered, image registration estimates the parameters of the geometrical transformation model that maps the sensed images back to its reference image. A feature-based approach to automated imageto-image registration is presented. The characteristic of this approach is that it combines Mexican-Hat Wavelet, Invariant Moments and Radon Transform. Feature Points from both images are extracted using Mexican-Hat Wavelet and controlpoint correspondence is achieved with invariant moments. After detecting corresponding control points from reference and sensed images, to recover scaling and rotation a line and triangle is form in both images respectively and applied radon transform to register images.
\end{abstract}

Keywords-Image Registration; Mexican-hat wavelet; Invariant Moments; Radon Transform.

\section{INTRODUCTION}

In this paper, we propose and implement a novel image registration method to register image with large rotation, scaling and translation. In all types of image registration, robustness of the algorithm is the main and required goal. However, due to diversification of images acquired their contents and purpose of their alignment, it is almost impossible to design universal method for image registration that fulfill all requirements and suits all types of applications [2]. Many of the image registration techniques have been proposed and reviewed [1], [2] [3]. Image registration techniques can be generally classified in two categories. Intensity based and feature based. The first category utilizes image intensity to estimate the parameters of a transformation between two images using an approach involving all pixels of the image. In second category a set of feature points extracted from an image and utilizes only these extracted feature points instead of all whole image pixels to obtain the transformation parameters. In this paper, a new algorithm for feature based image registration is proposed. The proposed algorithm is based on three main steps, feature point extraction, correspondence between extracted feature points and transformation parameter estimation.

Feature point and corner detectors have been area of interest to researcher in image registration. A point in an image is considered as a feature point if it has properties of distinctiveness and invariance. Several approach have been developed for feature point extraction and the points extracted by these methods differ in locations and structure, for example, edges, corner, blob-like shape, etc. In general, the main objective is to develop a feature point extractor that is robust to the most common geometric transformations and any possible degradation. Bas et al [4] compared major feature extraction techniques: Harris Corner detector, the Susan corner detector, and Achard-Rouquet detector. Feature extraction using Gabor wavelets have presented in [5], [6] give some good results in robustness to face recognition application. A popular method in computer vision applications is the scale invariant feature transform (SIFT) which is based on the feature point detection in scale space [7]. Recently developed technique which is based on scale interaction of Mexican hat wavelets [8]. This Mexican hat wavelet based feature extraction was inspired by the method presented in [8] [14].

We have used Mexican Hat Wavelet (MHW), to extract significant image features from reference and sensed image and make two sets of extracted feature points for both images. Next step of correspondence between extracted feature points is performed using Hu's Invariant moment-based similarity measure. This correspondence is evaluated using a circular neighborhood centered on each feature point. Among various types of moments available, Hu's moments is superior in terms of their orthogonality, rotation invariance, low sensitivity to image noise, fast computation and ability to provide faithful image representation [25]. After detecting matched feature points (control points), radon transform is used to estimate scaling and rotation. Radon transform gives $R\left(\theta, x^{\prime}\right)$ matrix from $f(x, y)$ matrix. The scaling factor has been obtained by getting ratio of maximum values of $R$ matrices of the images. Likewise the rotational difference is obtained by having $\theta$ difference between the matrices. After extracting these parameters derotation and descaling is performed to the sensed image. The translation difference is simultaneously found out by having coordinates of feature points. At last sensed image is shifted accordingly to fit and match with the reference image. Thus, the registered image is obtained and registration task is performed. Experimental results show that the proposed image registration algorithm leads to acceptable registration accuracy and robustness against several image deformations and image processing operations. 


\section{A. 2-D Continuous Wavelet Transform}

We can represent the 1-D continuous wavelet transform (CWT) in frequency domain given by following equation

$$
\mathrm{CWT}(s, \omega)=\sqrt{s} \mathrm{~F}(\omega) \Phi(s \omega)
$$

The 2-D CWT is the inner product of a signal or image with a scaled, rotated and translated version of a wavelet function. Now we represent 2-D continuos wavelet transform (CWT) in time domain and frequency domain by following equations

$$
\begin{array}{r}
\operatorname{CWT}(s, b, c)=\frac{1}{\sqrt{s}} \int \mathrm{f}(\mathrm{x}, \mathrm{y}) \psi\left(\frac{\mathrm{x}-b}{s}, \frac{\mathrm{y}-c}{s}\right) \mathrm{dxdy}, \\
\quad(\text { time domain) } \\
\operatorname{CWT}\left(s, \omega_{1}, \omega_{2}\right)=\sqrt{s} \mathrm{~F}\left(\omega_{1}, \omega_{2}\right) \Phi\left(s \omega_{1}, s \omega_{2}\right) \\
\text { (frequency domain) }
\end{array}
$$

The wavelet $\Psi$ is highly localized in space; it is either compactly supported or has fast decay. Its integral is zero: for a given scale $\mathrm{s}>0$ the CWT behaves like a band-pass filter, providing information on where in the image we can find oscillations or details at that scale. At small scales the CWT captures short-lived variations in color such as thin edges; comparing the CWT at different scales reveals what kind of discontinuity is present; at large scales it blurs the image. If the wavelet is stretched in one direction, the CWT gives information on local orientation in the image [16]. For our wavelet, we choose the Mexican hat wavelet, which is stretched in the direction of one of the axes in accordance with parameter [16].

$$
\operatorname{MHW}\left(x_{1}, x_{2}, s\right)=\frac{1}{\sigma}\left(2-\frac{x_{1}^{2}+x_{2}^{2}}{\sigma^{2}}\right) \mathrm{e}^{-\frac{x_{1}^{2}+x_{2}^{2}}{2 \sigma^{2}}}
$$

Where $\sigma=2^{-S}, S$ is a scale of the function, $x_{1}$ and $x_{2}$ are the vertical and horizontal coordinates respectively. When $\sigma=1$, MHW(s) is the laplacian of $\mathrm{g}\left(x_{1}, x_{2}\right)=\mathrm{e}^{-} 0.5\left(x_{1}^{2}+x_{2}^{2}\right)$, a bi dimensional Gaussian; it is isotropic, and in that case the CWT gives no information on object orientation. When scaled, its essential support is a disk with radius proportional to the scale. If $\sigma \neq 1$, we have the anisotropic Mexican hat, stretched out or shortened, and its support is an ellipse [17]. The frequency domain response of Mexican hat wavelet is given by

$$
\Phi\left(\omega_{1}, \omega_{2}\right)=-2 \pi\left(\omega_{1}^{2}+\omega_{2}^{2}\right) \mathrm{e}^{-\frac{1}{2}\left(\omega_{1}^{2}+\omega_{2}^{2}\right)}
$$

We observe the Mexican Hat shaped waveform in the time domain as shown in Fig. 1(a) and frequency domain in Fig. 1(b) respectively.

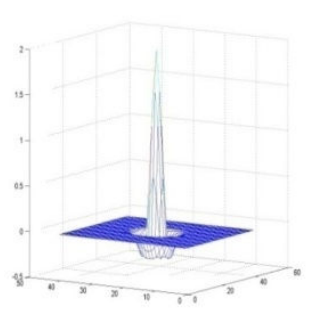

(a) Time domain

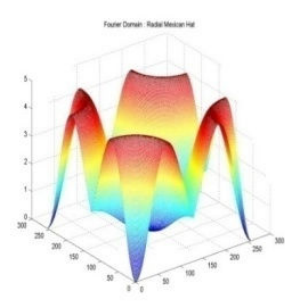

(b) Frequency domain

\section{B. MHW Response at Different Scale}

At lower scale, MHW give high frequency detail like corner, thin edges in the image. At higher scale, it gives low frequency smooth or blurs effect in the image. In order to extract feature point from the image, we have to decide appropriate scale. The Mexican Hat Wavelet has perfect circular symmetry in frequency and spatial domains. Fig.2 to Fig. 5 shows MHW response in time and frequency domain with coefficient of cameraman image at different scale 2, 34 , and 6 respectively.

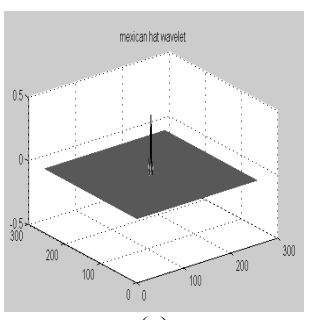

(a)

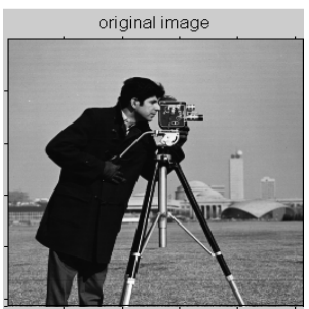

(c)

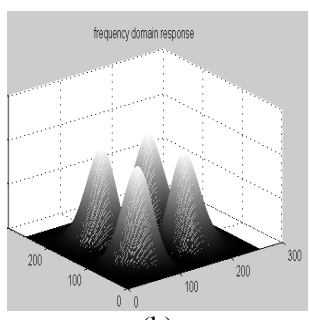

(b)

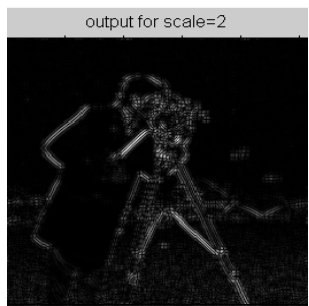

(d)
Figure 2 MHW (a) response in time domain (b) response in frequency domain (c) Cameraman image (d) MHW response of image at scale $=2$

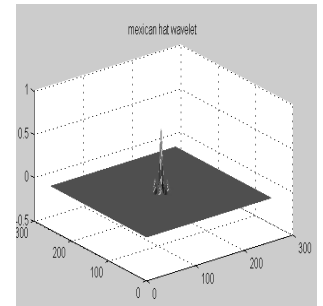

(b) $\mathrm{MHW}$ response of image at scale $=3$

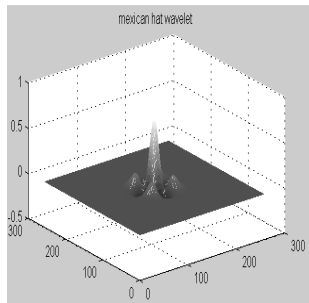

(a)

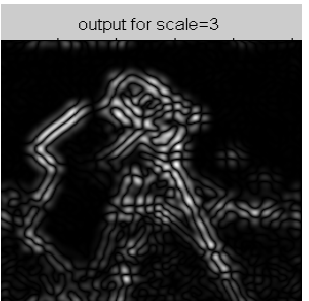

(a) (b)

(b)

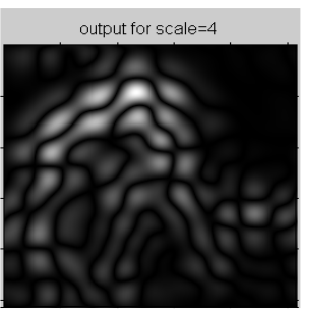

(b)
Figure 4 MHW responses (a) Time domain (b) MHW response of image at scale $=4$

Figure 1 Time and frqequecny domain representation of the 2D CWT [17]. 


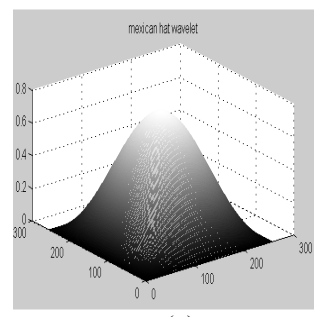

(a)

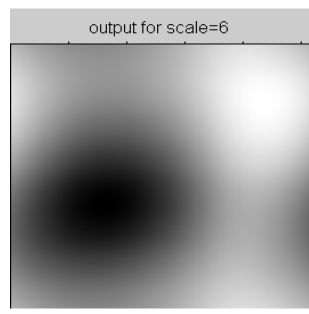

(b)
Figure 5 (a) MHW response in time domain

(b) MHW response of image at scale $=6$

From the above results, we can visualize that the Mexican-hat wavelet is equivalent to a band-pass filter. Tuning the wavelet scale controls the spread of the wavelet in the spatial domain and the bandwidth in the frequency domain. At scale 2, 3 and 4 MHW gives high frequency detail whereas at scale 6 and 9 it gives low frequency detail in the cameraman image. In our algorithm, we have decided to extract the feature points at scale 2, 3 and 4 .

\section{FEATURE Point EXtRaction USing ScAle INTERACTION OF MHW}

Our proposed algorithm is based on feature based image registration. So first and important task we have to do is feature extraction using scale interaction based on Mexican hat wavelets. For the image registration point of view, the extracted feature points must have to fulfill some basic objectives like they should be robust enough to some level of variations in scale and the feature extractor should have the ability to be modified to adapt image structures at different scales and the extracted features should have a well-localized support in the image. Here we have presented feature point extraction method that is based on scale interaction of Mexican hat wavelets. This method is based on finding the local maxima of the response of a feature detection operation, which involves convolving the image with the Mexican-hat wavelets. Feature point extraction using scale interaction of Gabor wavelets was proposed in [12]. The Mexican hat is a Laplacian of a Gaussian and its isotropic property makes it insensitive to orientation and a good choice for feature extraction [18]. A Mexican-hat wavelet has the shape of a signal with a positive peak in a negative dish. Convolving an image with Mexican-hat wavelets results in a response, which more likely detects blob-like shapes, bright areas surrounded by dark pixels or vice versa. Varying the width of the central peak of the Mexican-hat wavelet controls the size and the shape of the response [20] [23]. Mexican hat based feature extraction methods such as the ones in [19] and its further development in [21] [24].

An illustration of this feature extraction process is shown in Fig. 6. First of all reference image is taken as input, we call it $I_{R}(x, y)$. After taking its Fourier transform, its convolution is done with the Mexican hat wavelet. Here we use two different scales for the Mexican hat wavelet $\mathrm{S}_{1}$ and $\mathrm{S}_{2}$. This is known as
$\operatorname{MHW}\left(\mathrm{S}_{1}\right)$ and $\mathrm{MHW}\left(\mathrm{S}_{2}\right)$. After convolving the Mexican hat wavelet with the image and taking inverse Fourier transform, we obtain the response $R\left(S_{1}\right)$ and $R\left(S_{2}\right)$. This represented in the equation form as

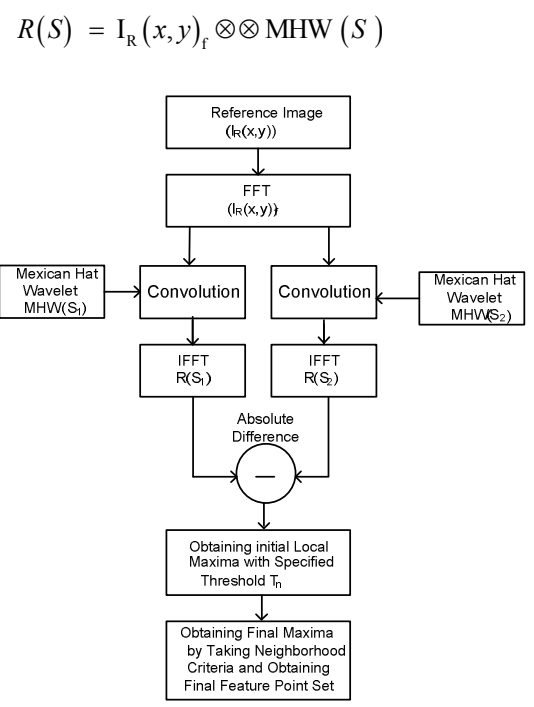

Figure 6 Block diagram of the feature extraction process.

Our next step is to get the scale interaction output. This is nothing but the absolute difference between the obtained responses. It is given as

$$
\begin{gathered}
\mathrm{R}\left(s_{1}, s_{2}\right)=\left|\mathrm{R}\left(s_{1}\right)-\mathrm{R}\left(s_{2}\right)\right| \\
=\left|\operatorname{IFFT}\left\{\mathrm{I}_{\mathrm{R}}(\mathrm{x}, \mathrm{y})_{\mathrm{f}} \otimes \otimes \mathrm{MHW}\left(\mathrm{S}_{1}\right)\right\}-\left\{\mathrm{I}_{\mathrm{R}}(\mathrm{x}, \mathrm{y})_{\mathrm{f}} \otimes \otimes \mathrm{MHW}\left(\mathrm{S}_{2}\right)\right\}\right|
\end{gathered}
$$

Where $I_{R}(x, y)_{f}$ denotes the Fourier transform of $I_{R}(x, y)$. The second stage of the feature extraction process localizes the feature points of the image by finding the local maxima of the response $R\left(S_{1}, S_{2}\right)$. This local maxima obtaining stage will be applied to the response $\mathrm{R}\left(\mathrm{S}_{1}, \mathrm{~S}_{2}\right)$, in Equation (8) using the following algorithm:

(i) Find the maximal values that are greater than the specified threshold $\mathrm{T}_{\mathrm{n}}$ in equally non-overlapped blocks of size $\mathrm{NxN}$; such initial maximal values may include points on the boundaries of the blocks, which do not represent a local maximum of $\mathrm{R}\left(\mathrm{S}_{1}, \mathrm{~S}_{2}\right)$.

(ii) Take each maximal point as the centre of a disk-shaped neighborhood of radius $r_{n}$ and find one local maximum in each neighborhood; this will eliminate maximal points that are not local maxima of $\mathrm{R}\left(\mathrm{S}_{1}, \mathrm{~S}_{2}\right)$ or local maxima that are too close to each other.

(iii) Repeat step (ii) until the obtained local maxima do not change locations.

(iv) In order to avoid the effects of the image borders on the feature extraction process, only the maxima found in the image area that is away from the image border by distance $r_{c}$ are kept. 


\section{A. Analysis of Feature point Extraction Process.}

Here we have taken an 8-bit gray level 'cameraman' image of size $256 \times 256$ as a reference image as shown in Fig. 7(a). First of all reference image and its convolution with the Mexican hat wavelet at scale $\mathrm{S}_{1}=2$ and $\mathrm{S}_{2}=4$ is taken which is shown in Fig. 7(b) and Fig. 7(c) respectively. Here inverted image of the result is shown only for the clear visualization purpose. The absolute difference between obtained responses is carried out as mentioned in Equation (7). The result of scale interaction process is shown in Fig. 7(d).

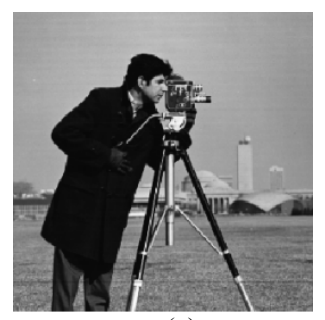

(a)

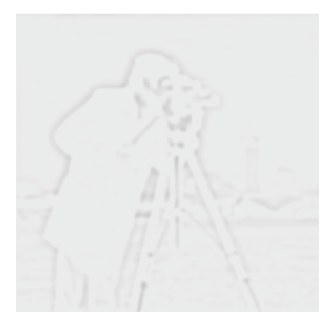

(c)

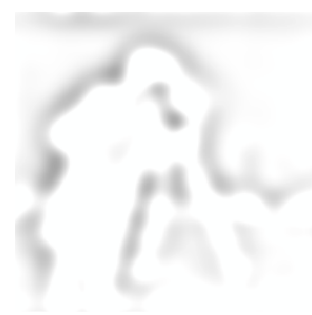

(b)

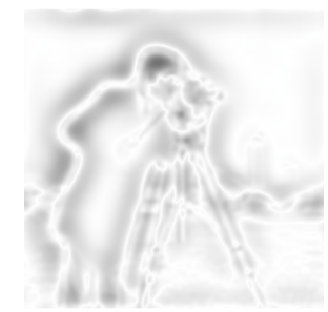

(d)
Figure 7 Feature point extraction stages (a) Reference image (b) Response of applying Mexican hat Wavelet with scale 2, (c) Response of applying Mexican-hat wavelet with scale 4, (d) Absolute difference of the two responses.

Next step is find the initial local maxima that are greater than a specified threshold $T_{n}=58$ in equally non overlapped blocks of size $32 \times 32$. Fig. 8 (a) shows the image with superimposed local maxima. After getting local maxima we can visualize that the obtained initial local maxima might not be actual peaks and some of them might be very close to each other and looks like bunch of feature points. Take each local maximum point as the center of a disk-shaped neighborhood of radius $r_{n}$ is 16 and find one local maximum in each neighborhood, this will eliminate local maxima that are very close to each other. Repeat above step until the obtained local maxima do not change locations. The final extracted feature points shown in Fig. 8 (b).

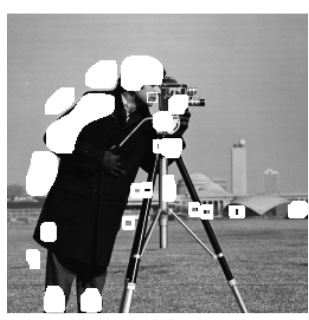

(a)

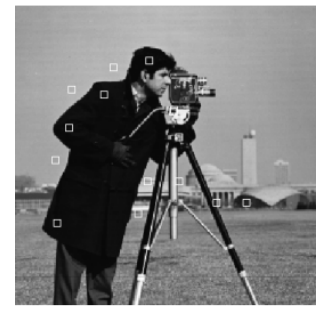

(b)
Figure 8 Reference image with the extracted points superimposed on the image (a) after Thresholding (b) after applying disk shaped neighborhood criteria.
We will get the similar results for the sensed image, represented in Fig. 9 and Fig. 10.

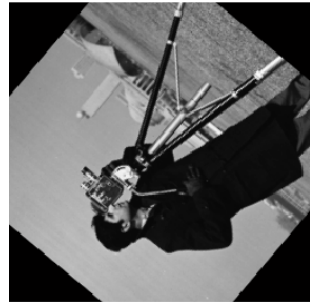

(a)

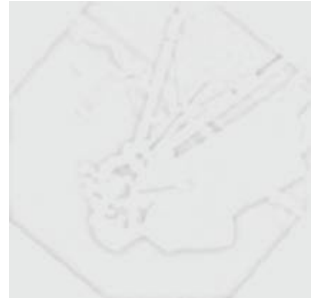

(c)

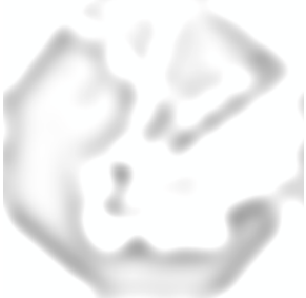

(b)

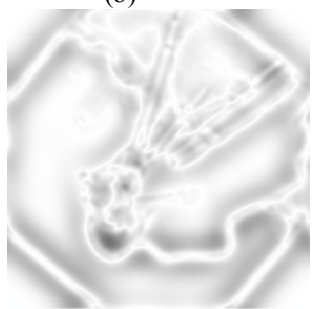

(d)
Figure 9 Feature point extraction stages (a) Sensed image (b) Response of MHW with $\mathrm{S}=2$ (c) Response of applying MHW with scale $=4$ (d) Absolute difference of the two responses.

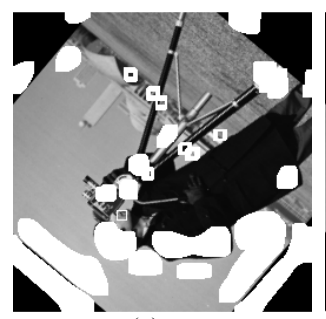

(a)

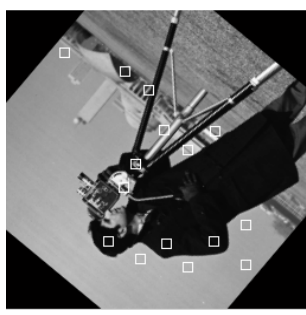

(b)
Figure 10 Sensed image with the extracted points superimposed on the image (a) after Thresholding (b) after applying disk shaped neighborhood criteria.

\section{B. Performance Analysis}

The performance of this Mexican hat wavelet based feature point extractor is evaluated by taking one image as reference and applying different types of degradations to it and using it as sensed image. After visualization of the extracted feature points from both of these images as shown in Fig.11, we can say that it is a robust feature point extractor which extracts those feature points which are as many as possible in the same, or within the neighborhood of locations, with respect to the contents of the image, regardless of any degradations or distortions applied on the image. The presented scaleinteraction Mexican hat wavelets feature point extraction method will be used in image registration as discussed in the subsequent chapters.

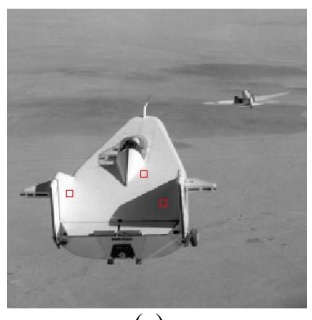

(a)

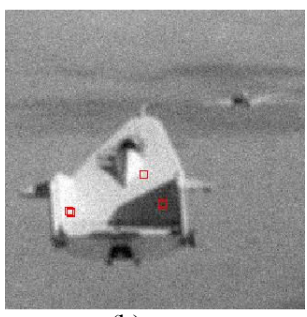

(b) 


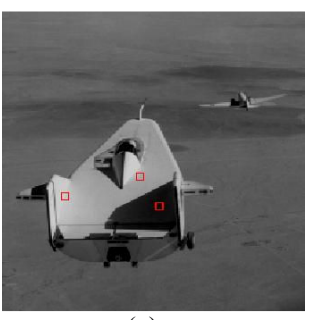

(c)

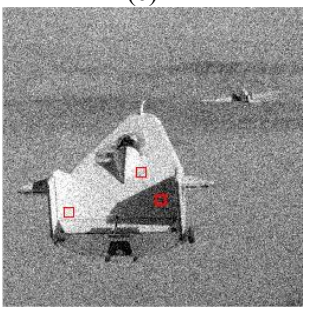

(e)

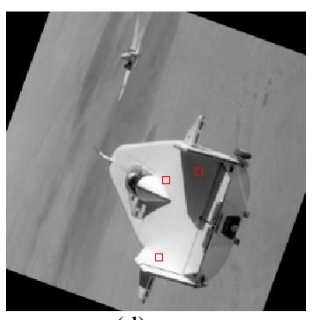

(d)

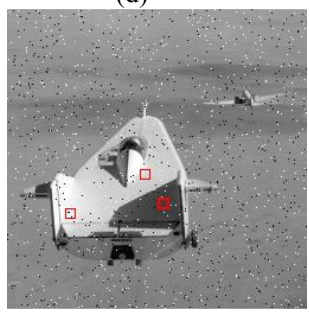

(f)
Figure 11 Feature point extraction of distorted/degraded images: (a) No distortion, (b) Blurring, (c) Brightness change, (d) Rotation, (e) Gaussian noise contamination (f) 'Salt and Pepper' noise

\section{FEATURE POINT MATCHING USING INVARIANT MOMENTS}

Feature Points from both images are extracted using Mexican-Hat Wavelet and control-point correspondence is achieved with invariant moments. After detecting corresponding control points from both images radon transform approach is applied to register images. One problem in image registration is automatic registration of an image regardless of its size, position and orientation. To achieve this goal, the extracted image features should have the invariance properties against image transformations including scale change, translation and rotation [25]. These image transformations can he approximated by affine transformation which is an important class of linear 2-D geometric transformation. The affine transformation preserves collinearity (i.e., all points lying on a line initially still lie on a line after transformation) and ratios of distances (e.g., the midpoint of a line segment remains the midpoint after transformation). The image features with the invariance properties are called image invariants.

Fourier descriptors and Hu's seven moment invariants are the most popular shape based image invariants and have been used in image recognition, indexing, and retrieval systems [13],[15][26]. Fourier descriptors are boundary-based image features which only compute the pixels along the image contours. On the contrary, Hu's seven moment invariants are region-based image features which take all of the pixels of the image into account [15]. Hu's seven moment invariants have the invariance property against affine transformations including scale change, translation and rotation.

\section{A. Moment Representation}

Moment invariants (MIs) are a class of image descriptors first derived by $\mathrm{Hu}$ [25], [26] who employed the results of the theory of algebraic invariants and derived his seven famous invariants to rotation of 2-D objects. Moment-based invariants are the most common region-based image invariants which have been used as pattern features in many applications [27].
$\mathrm{Hu}$ first introduced a set of invariants using nonlinear combinations based on regular moments in 1961 [25]. For a 2D continuous function $f(x, y)$, the moment of order $(\mathrm{p}+\mathrm{q})$ is defined as

$$
m_{p q}=\int_{-\infty}^{\infty} \int_{-\infty}^{\infty} x^{p} y^{q} f(x, y) d x d y \text { for } p, q=0,1,2, \ldots \ldots .
$$

A uniqueness theorem states that if $f(x, y)$ is piecewise continuous and has nonzero values only in a finite part of the $x y$-plane, moment of all orders exist, and the moment sequence $\left(m_{p q}\right)$ is uniquely determined by $f(x, y)$. Conversely, $\left(m_{p q}\right)$ is uniquely determined by $f(x, y)$. The central moments are defined as

$$
\mu_{p q}=\int_{-\infty}^{\infty} \int_{-\infty}^{\infty}(x-\bar{x})^{p}(y-\bar{y})^{q} f(x, y) d x d y
$$

$$
\text { where } \bar{x}=\frac{m_{10}}{m_{00}} \quad \text { and } \bar{y}=\frac{m_{01}}{m_{00}}
$$

If $f(x, y)$ is a digital image, then equation (5.2) becomes

$$
\mu_{p q}=\sum(x-\bar{x})^{p}(y-\bar{y})^{q} f(x, y)
$$

and the normalized central moments, denoted $\eta_{p q}$, are defined as

$$
\eta_{p q}=\frac{\mu_{p q}}{\mu_{00}^{\gamma}}, \text { where } \gamma=\frac{p+q}{2}+1 \text { for } \mathrm{p}+\mathrm{q}=2,3 \ldots \ldots \ldots
$$

A set of seven invariant moments can be derived from the second and the third moments by $\mathrm{Hu}[20]$. As shown below, $\mathrm{Hu}$ derived the expressions from algebraic invariants applied to the moment generating function under a rotation transformation. They consist of groups of nonlinear centralized moment expressions. The result is a set of absolute orthogonal moment invariants, which can be used for scale, position, and rotation invariant pattern identification.

$$
\begin{aligned}
\phi_{1}= & \eta_{20}+\eta_{02} \\
\phi_{2}= & \left(\eta_{20}-\eta_{02}\right)^{2}+4 \eta_{11}^{2} \\
\phi_{3}= & \left(\eta_{30}-3 \eta_{12}\right)^{2}+\left(3 \eta_{21}-3 \eta_{03}\right)^{2} \\
\phi_{4}= & \left(\eta_{30}+\eta_{12}\right)^{2}+\left(\eta_{21}+\eta_{03}\right)^{2} \\
\phi_{5}= & \left(\eta_{30}-3 \eta_{12}\right)\left(\eta_{30}+\eta_{12}\right)\left[\left(\eta_{30}+\eta_{12}\right)^{2}-3\left(\eta_{21}+\eta_{03}\right)^{2}\right] \\
\phi_{6}= & \left(\eta_{20}-\eta_{02}\right)\left(\eta_{30}+\eta_{12}\right)\left[\left(\eta_{30}+\eta_{12}\right)^{2}-\left(\eta_{21}+\eta_{03}\right)^{2}\right]+ \\
& 4 \eta_{11}\left(\eta_{30}+\eta_{12}\right)\left(\eta_{21}+\eta_{03}\right) \\
\phi_{7}= & \left(3 \eta_{21}-\eta_{03}\right)\left(\eta_{30}+\eta_{12}\right)\left[\left(\eta_{30}+\eta_{12}\right)^{2}-3\left(\eta_{21}+\eta_{03}\right)^{2}\right] \\
& +\left(3 \eta_{12}-\eta_{30}\right)\left(\eta_{21}+\eta_{03}\right)\left[3\left(\eta_{30}+\eta_{12}\right)^{2}-\left(\eta_{21}+\eta_{03}\right)^{2}\right]
\end{aligned}
$$

\section{Proposed Automatic IMAge REgistration ALGORITHM}

This Section introduces an algorithm for automatic feature based image registration. The main objective of the proposed algorithm is accurately registering images which are geometrically distorted. The proposed algorithm is shown in Fig. 12. 


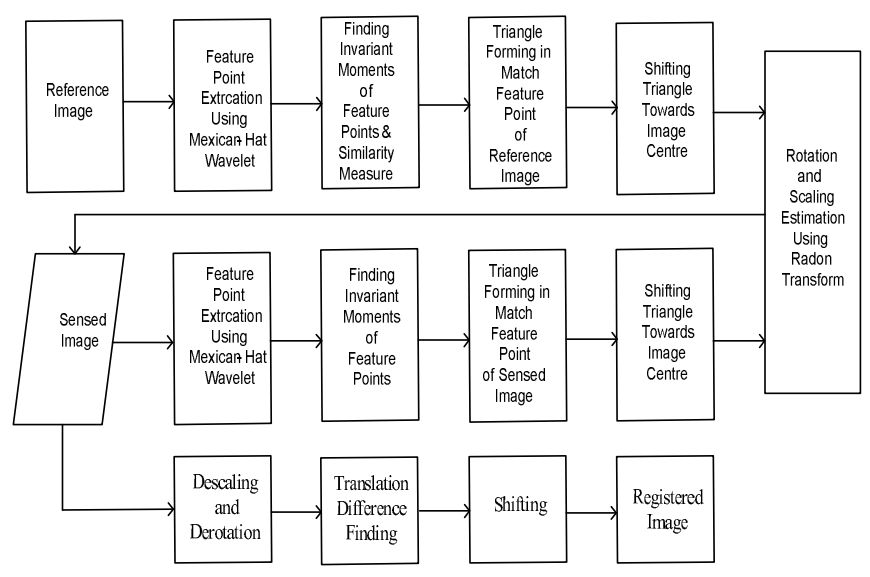

Figure 12 Proposed Automatic Image Registration Algorithm

As shown in Fig. 12, automatic feature points are extracted using Mexican-Hat Wavelet as discussed in Section III. After the strong feature points extracted from reference and sensed images, a correspondence mechanism is required between these two feature point sets. This correspondence mechanism fulfils the requirement of pairing the feature point of reference image with its correspondent one in the sensed image. In this proposed algorithm, Invariant moment based similarity measure approach is used to establish the correspondence between the two images.

This correspondence is evaluated using a circular neighbourhood cantered on each and every feature point. For every extracted feature point, select a circular neighbourhood of radius $\mathrm{R}$ centred at this point and construct a set of absolute orthogonal moment invariants vectors $\left(\phi_{1}, \phi_{2}, \phi_{3}, \ldots \ldots \ldots \phi_{7}\right)$ as described in Equations (12) to (18). The similarity between the regions is judged by computing the Euclidean Distance between invariable regions of reference image with sensed image.

In our proposed method, we had calculated distance between feature point moment invariant vectors of reference image and moment invariant vectors of all feature points of sensed image. For example, suppose there are five features point in reference image and seven feature points in sensed image. We had considered any one feature point invariant moment vector and find the distance between all extracted feature points invariant moment vectors of sensed image. Then we had considered second feature point moment invariant vector and find the distance with all extracted feature points vectors of sensed image. After detecting distance, corresponding feature points are detected by minimum distance rule with the threshold in the Euclidean space of the invariants.

After detecting, similar feature points from both images, we had considered any three similar feature points as control points from both images and form a triangle by considering any one feature point and find the maximum distance across all feature points in the image. Then, draw a line between this maximum distance feature points and second line with maximum slope and connect third line to form a triangle. This way, we had drawn triangle in both images and shift it towards image centre.

After this, we had applied radon transform algorithm for extracting scaling. The radon function computes projections of an image matrix along specified directions. A projection of a two-dimensional function $f(x, y)$ is a set of line integrals. The radon function computes the line integrals from multiple sources along parallel paths, or beams, in a certain direction [10] [13]. The beams are spaced 1 pixel unit apart. Radon transform of a two dimensional function $f(x, y)$ is defined as

$$
\begin{aligned}
& R(r, \theta)[f(x, y)]=\int_{-\infty}^{+\infty} \int_{-\infty}^{+\infty}(f(x, y) \delta(r-x \cos \theta- \\
& y \sin \theta)) d x d y \quad(19)
\end{aligned}
$$

Where, $r$ is the perpendicular distance of a line from the origin and $\theta$ is the angle formed by the distance vector.

\section{A. Analysis of Proposed Image Registration Algorithm}

In this section, the performance evaluation is done for the proposed automatic image registration algorithm, for different types of distortions. An original image or reference image is geometrically distorted and different types of geometrical deformations added in it. A set of experiments has been performed to evaluate the performance of the proposed algorithm with respect to registration accuracy and robustness.

(i) Feature Point Extraction Using Mexican-Hat Wavelet: Here we select an 8-bit gray level 'living room' image as a reference image. The size of the image is $256 \times 256$. Fig. 13 (a) and (b) shows the reference and sensed image respectively. Fig. 13 (a) and (b) shows the final extracted feature points $\left(R_{1}, R_{2}, \ldots . R_{5}\right)$ in reference image and $\left(S_{1}\right.$, $\mathrm{S}_{2}, \ldots . \mathrm{S}_{5}$ ) in sensed image.

(ii) After extracting feature points from both images, we determined invariant moments by considering circular template across all feature points. We considered all the points of circular neighborhood of radius 20 around feature points. Table-I and Table-II shows invariant moments of extracted features of reference image and sensed image. Fig. 14 \& Fig. 15 shows circular neighborhood around all extracted feature points of images.

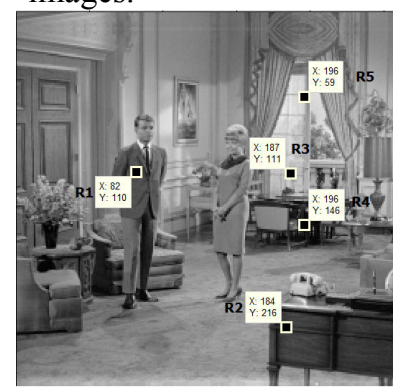

(a)

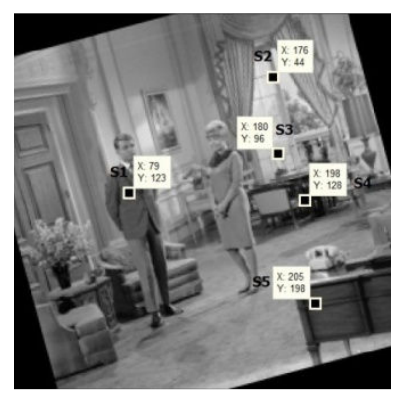

(b)
Figure 13 (a) Reference image (b) Sensed Image with feature points (Rotation $=15^{0}$, Scaling $\left.=1\right)$ 
TABLE I. INVARIANT MOMENT OF REFERENCE IMAGE FEATURE POINTS

\begin{tabular}{|c|c|c|c|c|c|c|c|}
\hline Moment & $\phi_{1}$ & $\phi_{2}$ & $\phi_{3}$ & $\phi_{4}$ & $\phi_{5}$ & $\phi_{6}$ & $\phi_{7}$ \\
\hline MR1 & 2.7121 & 8.4255 & 11.0956 & 10.7246 & 21.6372 & 14.9400 & 22.6079 \\
\hline MR2 & 2.6609 & 7.6623 & 9.7978 & 9.9937 & 19.9234 & 13.8302 & 20.3094 \\
\hline MR3 & 3.0514 & 9.3793 & 11.442 & 11.879 & 23.8940 & 16.5696 & 23.5881 \\
\hline MR4 & 2.5252 & 7.6820 & 9.0593 & 9.4312 & 18.6784 & 13.3216 & 19.7068 \\
\hline MR5 & 3.0817 & 9.2234 & 12.446 & 12.419 & 25.0066 & 17.0800 & 24.9986 \\
\hline
\end{tabular}

TABLE II. INVARIANT MOMENT OF SENSED IMAGE FEATURE POINTS

\begin{tabular}{|c|c|c|c|c|c|c|c|}
\hline Moment & $\phi_{1}$ & $\phi_{2}$ & $\phi_{3}$ & $\phi_{4}$ & $\phi_{5}$ & $\phi_{6}$ & $\phi_{7}$ \\
\hline MS1 & 2.7095 & 8.3341 & 11.2460 & 10.7846 & 21.9022 & 14.9727 & 22.0125 \\
\hline MS2 & 3.08188 & 9.3097 & 12.4795 & 12.4058 & 24.9254 & 17.1700 & 25.1113 \\
\hline MS3 & 3.05326 & 9.1451 & 11.5194 & 11.9435 & 23.8650 & 16.5162 & 23.7922 \\
\hline MS4 & 2.52788 & 7.6959 & 9.08295 & 9.39771 & 18.6405 & 13.2671 & 19.6062 \\
\hline MS5 & 2.66365 & 7.6283 & 9.79171 & 9.97759 & 19.8722 & 13.8206 & 20.5355 \\
\hline
\end{tabular}

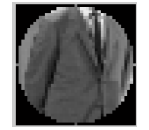

(a)

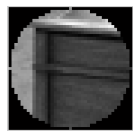

(b)

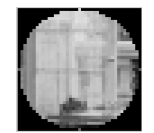

(c)

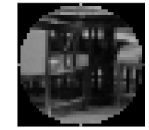

(d)

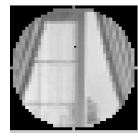

(e)
Figure 14 circular neighborhood around feature points (a) R1 (b) R2 (c) R3 (d) R4 (e) R5

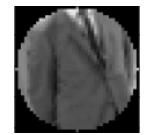

(a)

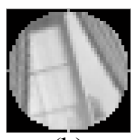

(b)

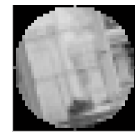

(c)

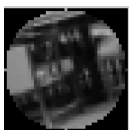

(d)

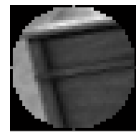

(e)
Figure 15 circular neighborhood around feature points (a) S1 (b) S2 (c) S3 (d) S4 (e) S5

(iii) After this, we find distance between invariant moments of reference and sense feature points. First, we consider moment invariants vector MR1 and determined the distance with all moment invariant vectors (MS1, MS2,....MS5) of sensed image. The minimum distance between this is mark as a corresponding feature point. Table-III shows the distance calculation of reference feature points and sensed feature points. As shown in above Table-III, the minimum distance indicate the similar feature points in reference and sensed image. The minimum distance $\mathrm{D}_{\mathrm{MR} 1 \mathrm{MS} 1}$ indicate feature point $\mathrm{R} 1$ in reference image is match with feature point $\mathrm{S} 1$ of sensed image.

TABLE III. MINIMUM DISTANCE CALCULATION

\begin{tabular}{|c|c|c|c|c|c|}
\hline Distance & D $_{\text {MRIMS1 }}$ & $\mathrm{D}_{\text {MR1MS2 }}$ & $D_{\text {MRIMS3 }}$ & $\mathrm{D}_{\text {MRIMS4 }}$ & $\mathrm{D}_{\text {MR1MS5 }}$ \\
\hline & 1.1975 & 12.3404 & 7.6915 & 11.9250 & 7.8536 \\
\hline Distance & $\overline{\overline{D_{M R 2 M S 1}}}$ & $\mathrm{D}_{\mathrm{MR} 2 \mathrm{~N}}$ & $\mathrm{D}_{\mathrm{MR} 2 \mathrm{MS} 3}$ & $\mathrm{D}_{\mathrm{MR} 2 \mathrm{MS} 4}$ & $D_{\text {MR2MS5 }}$ \\
\hline & 5.1995 & 20.3057 & 15.6568 & 4.0268 & 0.3459 \\
\hline Distance & $\overline{D_{M R 3}}$ & $\mathrm{D}_{\mathrm{MR} 31}$ & & $\mathrm{D}_{\mathrm{MR} 3}$ & \\
\hline & 10.4268 & 4.8187 & 0.6637 & 19.5860 & 15.5146 \\
\hline Distance & $\mathrm{D}_{\mathrm{MR} 4}$ & $\mathrm{D}_{\mathrm{MF}}$ & & & \\
\hline & & 24.0790 & 19.4301 & 0.2666 & 3.9924 \\
\hline Distance & & $\mathbf{D}_{\mathrm{MI}}$ & & $\overline{D_{M R}}$ & \\
\hline & 14.8786 & 0.4166 & 4.4214 & 24.0379 & 19.9665 \\
\hline
\end{tabular}

(iv) Then, we form a triangle between any three similar feature points in reference image $(\mathrm{R} 1, \mathrm{R} 2, \mathrm{R} 5)$ and sense image $(\mathrm{S} 1, \mathrm{~S} 2, \mathrm{~S} 5)$ and apply radon transform for calculating scaling between the images registering the images.
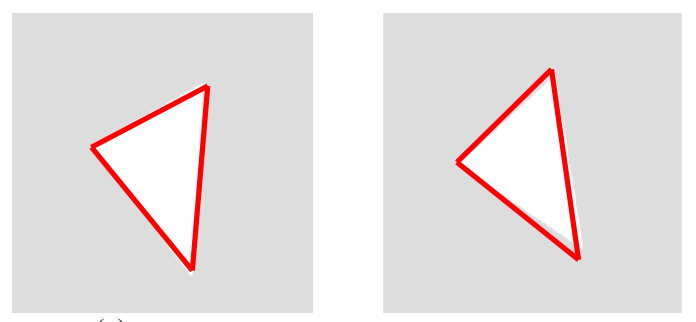

(a)

Figure 16 Triangle after region filling for (a) reference image

(b)

Radon transform is now applied on Fig. 16. Two matrices are obtained after applying radon transform on Fig. 16(a) and Fig. 16(b). Practical value of $R_{1 \max }$ is 155.29 for reference image and $R_{2 \max }$ is 154.49 for sensed image. The ratio $R_{2 \max } / R_{1 \max }$ gives 0.995 which is nothing but the desired and accurate scaling between sensed and reference image. In order to recover rotation between images, we have formed line between two similar feature points. To make a line we need to get all the points which satisfy the line equation. We get floating point numbers so approximation is done and nearest integer is chosen to convert them to integers. After making line the images look like Fig. 17.

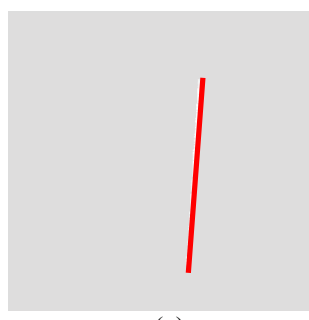

(a)

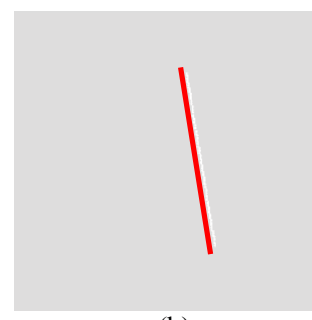

(b)

Figure 17 Line formed after joining two points are for (a) reference image and (b) sensed image.

Radon transform is now applied on Fig. 17. Here, column size is $360^{\circ}$ because the radon transform is applied for $1: 360^{\circ}$. Now cross-correlation is obtained between this and rotation is extracted. The maximum value of correlation occurs at the coordinates $[\mathrm{r}, \mathrm{c}]=[405,345]$. Here we want correlation of the column as shifting operation is related with $\theta$. Maximum column size of template i.e. $360^{\circ}$ is subtracted from c, therefore $360^{\circ}$ $345^{\circ}=15^{\circ}$. This is nothing but extracted rotation of sensed image with respect to reference image.

(v) As we know the four steps of image registration, three steps are performed in previous chapters. The last step is image resampling and transformation. Here descaling, derotation and translation is applied to sensed image in order to obtain registered image. The final registered image is shown in Fig. 18. 


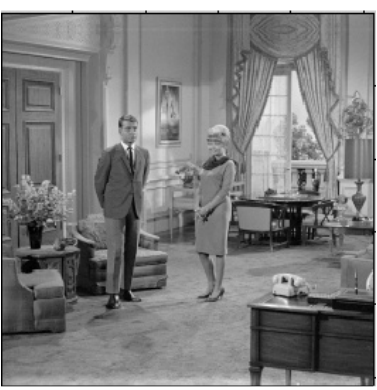

(a)

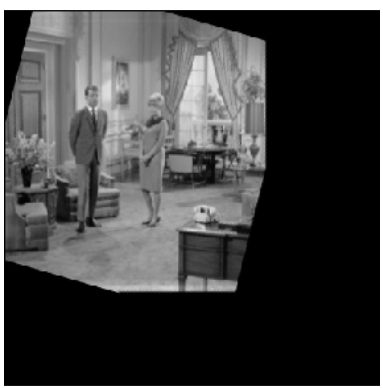

(c)

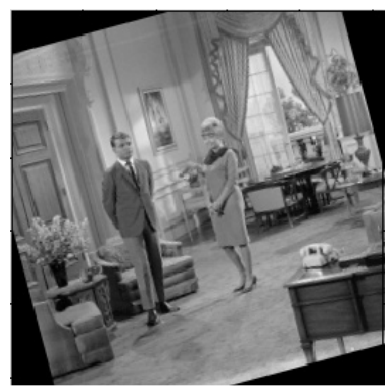

(b)

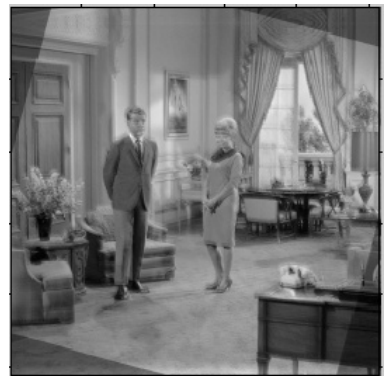

(d)
Figure 18 (a) Reference Image (b) Sensed image with rotation $15^{0}$ (c) Registered image (d) Sensed image overlaid on reference image

\section{B. Simulation Results}

Here we have considered different 8-bit gray level images as a reference image and sensed image with geometrical deformation. In Fig. 19(b), the sensed image having $30^{\circ}$ rotation, scale $=1.3$. After applying proposed algorithm, the registered image is shown in Fig. 19(d). It shows the accuracy of image registration. After successful registration of the "living room" image, we have applied different geometrical deformations. Fig. 20 to Fig. 22 shows the registered images with different geometrical deformations.

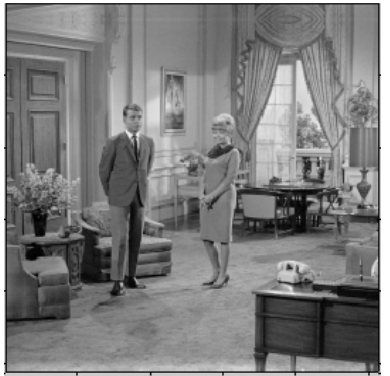

(a)

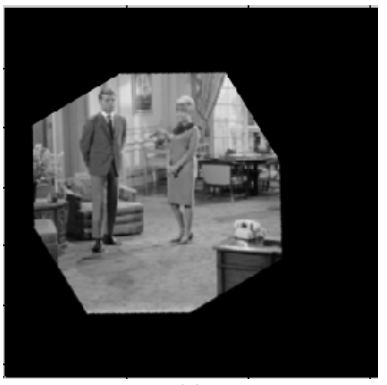

(c)

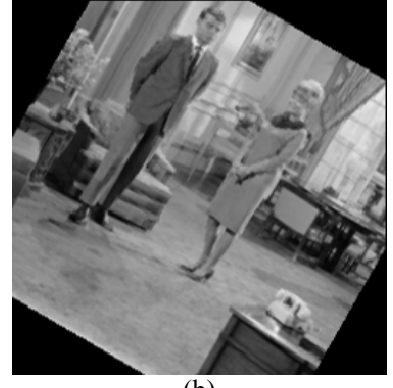

(b)

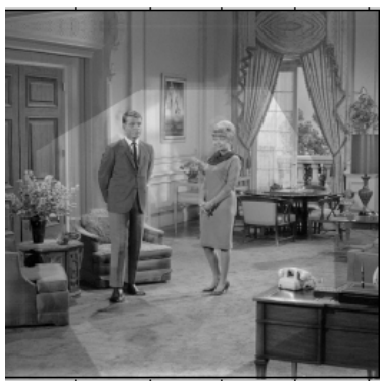

(d)
Figure 19 (a) Reference Image (b) Sensed image with rotation $30^{\circ}$, scale $=1.3$ (c)Registered image (d) Sensed image overlaid on reference image

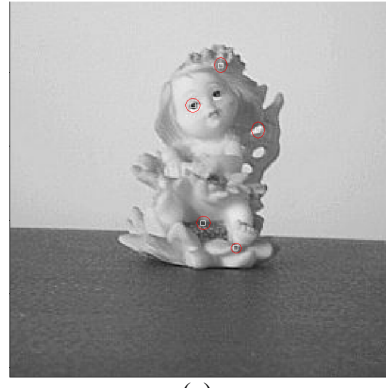

(a)

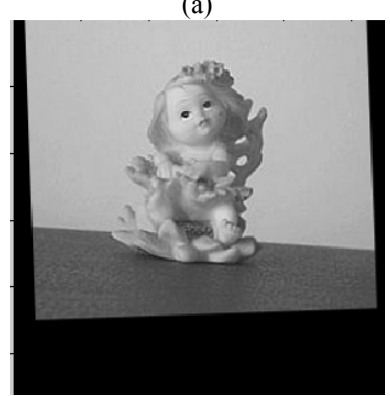

(c)

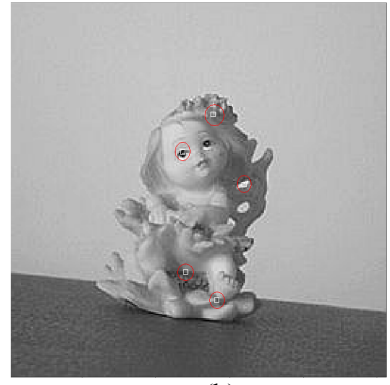

(b)

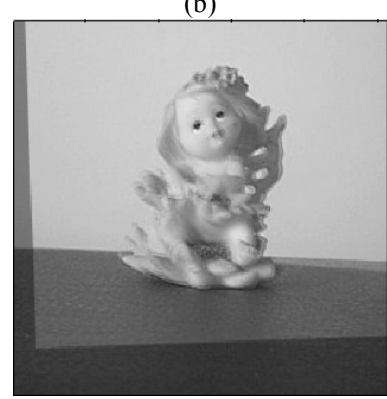

(d)
Figure 20 (a) Reference Image (b) Sensed image with rotation $2^{0}$, scale $=1$ (c) Registered image (d) Sensed image overlaid on reference image
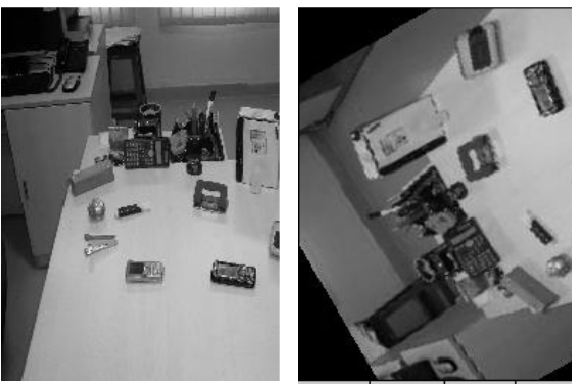

(a)

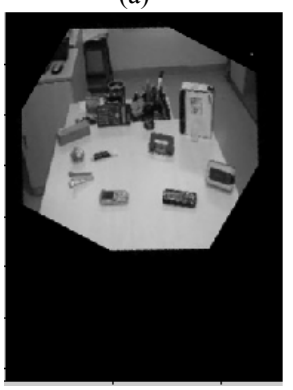

(c)

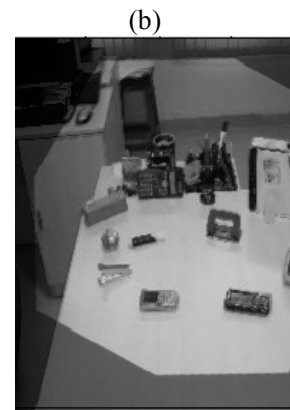

(d)
Figure 21(a) Reference Image (b) Sensed image with rotation $240^{\circ}$, scale $=1.2$ (c)Registered image (d) Sensed image overlaid on reference image

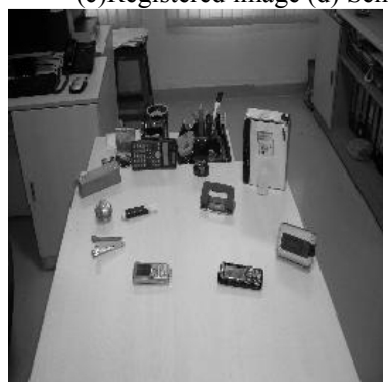

(a)

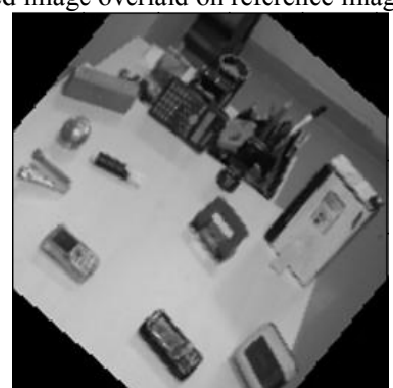

(b) 


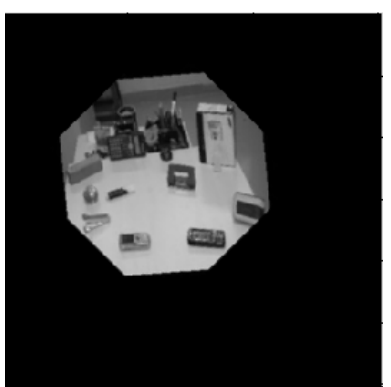

(c)

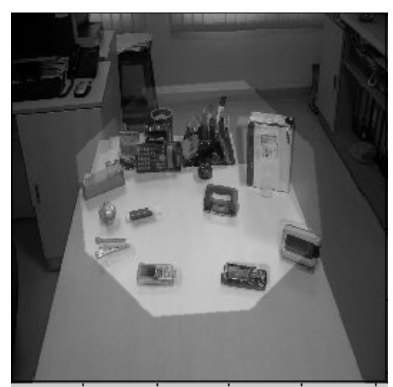

(d)
Figure 22 (a) Reference Image (b) Sensed image with rotation $45^{\circ}$, scale $=1.8$ (c)Registered image (d) Sensed image overlaid on reference image

\section{CONCLUSIONS}

In this paper, a new feature based image registration approach is proposed. This type of approach gives a new dimension to the existing feature based image registration methods. After successful feature extraction using Mexican Hat Wavelet, correspondence between extracted feature points is established using Hu's invariant moments. Once the set of correctly matched feature point pairs between two images are found, the scaling, and rotations are extracted using radon transform. With this proposed algorithm, we registered images with any degree of rotation and scale up to 1.8 .

\section{REFERENCES}

[1]. L.G. Brown, "A survey of Image Registration Techniques," $A C M$ Computing Surveys, vol. 24, no. 4, pp. 325-3760, 1992.

[2]. Gang Hong and Yun Zhang, "Combination of feature-based and areabased image registration technique for high resolution remote sensing image," Geoscience and Remote Sensing Symposium, 2007, IGARSS 2007, pp.377-380, 2007.

[3]. B. Zitova and J. Flusser, "Image Registration methods: A Survey," Image Vision Computing, vol. 21, no. 11, pp. 977-1000, 2003.

[4]. P.Bas, J-M.Chassery, B.Macq, "Geometrically invariant watermarking using feature points, IEEE Trans. On Image Processing," Vol.11,pp. 1014-1028,2002

[5]. K.vinay and B.Shreyas, "Face recognition using gabor wavelets," in Fortieth Asilomar Conference on Signal, Systems and Computers,2006, ACSSC'06, pp.593-597,2006

[6]. G.Wang and Z.Ou, "Face recognition based on image enhancement and gabor feature," in Proc. The Sixth World Congress on Intelligent Control and Automation,2006, WCICA 2006, Vol. 2,pp.97619764,2006

[7]. D.G.Lowe, “ Distinctive image features from scale invariant key points," International Journal of Computer Vision, Vol. 60,pp.91110,2004

[8]. Steven Gillan and Pan Agathoklis, "Image registration using feature points, Zernike moments and an M-estimator," The International Midwest Symposium on Circuits and Systems (MWSCAS) is the oldest Circuits and Systems symposia, Washington, pp.434-437 August 2010.

[9]. Collins, D.L. Holmes, C.J. Peters, T.M. and Evans, A.C., "Automatic 3D model-based neuro anatomical segmentation". Human Brain Mapping, pp.190-208, 1995.

[10] M.Van Ginkel, C.L.Luengo Hendriks and L.J. van Vliet, “ A short introduction to the radon and hough transforms and how they relate to each other," in the Quantitative Image Group Technical Report Series, N. QI-2004-01, pp.1-9,2004
[11] K. P. Soman, K. I. Ramachandran, "Insight into Wavelets, from theory to practice, Prentice-Hall of India Private Limited, 2006.

[12] B. Manjunath, C. Shekhar, and R. Chellappa, "A new approach to image feature detection with applications," Pattern Recognition, vol. 29, No. 4, pp. 627-640, 1996.

[13] A K Jain, "Digital Image Processing," $2^{\text {nd }}$ Edition, Prentice Hall Publication, 2006.

[14] M. S. Yasein and P. Agathoklis, A robust, feature-based algorithm for aerial image registration," in Proceedings of the IEEE International Symposium on Industrial Electronics (ISIE 2007), Vigo, Spain, pp. 1731-1736, 2007.

[15] R C Gonzalez, "Digital Image Processing Using MATLAB," $2^{\text {nd }}$ Edition, Mc-Graw Hill Publication, 2010.

[16] Heejong Yoo, "2D Continuous Wavelet Transform", Available on line on http://www.isye.gatech.edu., Downloaded in September 2010.

[17] Diego Romero, Ana Ruedin, and Leticia Seijas, Wavelet-Based Feature Extraction for Handwritten Numerals, in Springer Berlin / Heidelberg, Image Analysis and Processing (ICIAP 2009), vol. 5716, pp. 374-383, 2009.

[18] S. Bhattacharjee and M. Kutter, "Compression tolerant image authentication," in Proceedings of the IEEE International Conference on Image Processing (ICIP-1998), Chicago, USA, pp. 435-439, 1998.

[19] M. Kutter, S. K. Bhattacharjee, and T. Ebrahimi, Toward second generation watermarking schemes," in Proceedings of the IEEE International Conference on Image Processing (ICIP-1999), Kobe, Japan, pp. 320-323, 1999.

[20] M. S. Yasein and P. Agathoklis, Automatic and robust image registration using feature points extraction and zernike moments invariants," in Proceedings of the Fifth IEEE International Symposium on Signal Processing and Information Technology, Athens, Greece, pp. 566-571,2005.

[21] M. S. Yasein and P. Agathoklis, A robust, feature-based algorithm for aerial image registration," in Proceedings of the IEEE International Symposium on Industrial Electronics (ISIE 2007), Vigo, Spain, pp. 1731-1736, 2007.

[22] Chih-Wei Tang and Hsueh-Ming Hang, A Feature-Based Robust Digital Image Watermarking Scheme, in IEEE Trans. on Signal Processing, vol. 51, No.4, pp.950-959, 2003.

[23] D. Marr and E. Hildreth, "Theory of edge detection," Proceedings Royal Society of London, vol. 207, No. 1167, pp. 187-217, 1980.

[24] A.L. Cunha, J. Zhou, and M.N. Do, "The Nonsubsampled Contourlet Transform: Theory, Design, and Applications," IEEE Trans. Image Processing, vol. 15, No. 10, pp. 3089-3101, 2006.

[25] Zhihu Huang and Jinsong Leng, "Analysis of Hu's Moment Invariants on Image Scaling and Rotation," $2^{\text {nd }}$ International Conference on Computer Engineering and Technology, Vol.7, pp.476-480, 2010.

[26] Qing Chenl, Petriul, and Xiaoli Yang, "A Comparative Study of Fourier Descriptors and Hu's Seven Moment Invariants for Image Recognition," Canadian Conference on Electrical and Computer Engineering, pp.103106, 2004.

[27] Youcef Bentoutou and N. Taleb, "Automatic Extraction of Control Points for Digital Subtraction Angiography Image Enhancement," IEEE Trans. on nuclear science, Vol. 52, No. 1, pp.238-246, 2005. 


\section{AUTHORS PROFILE}

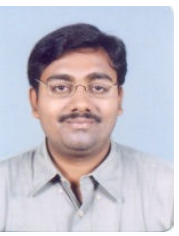

Prof. Jignesh N. Sarvaiya received his M.Tech. Degree from IIT Bombay, India. Presently, He is Assistant Professor at Electronics Engineering. Department, SVNIT Surat, (Gujarat) India. His Research Interest includes Image Processing, Image Registration, Bio-Medical Instrumentation and Electronics System Design.

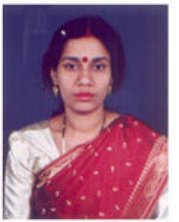

Dr. Suprava Patnaik received M.Tech. Degree in Electronic \& Communication Engineering from NIT, Rourkela, in 1992, and Ph.D. from Electrical, Electronics \& Communication Engineering Deprtment, IIT, Kharagpur in 2004. Presently, She is Associate Professor in the Electronics Engineering Department, SVNIT, Surat (Gujarat) India. Her research interests include Signal and Image processing, Image Segmentation, Pattern Recognitation, Digital Watermarking, Image Registration and Video Compression . 